\title{
Supplementary Information for Topological Simplification of Complex Knots Untied in Elongational Flows
}

\author{
Beatrice W. Soh ${ }^{1}$, Alexander R. Klotz ${ }^{2}$, and Patrick S. Doyle*1 \\ ${ }^{1}$ Department of Chemical Engineering, Massachusetts Institute of Technology, Cambridge, \\ Massachusetts 02139, United States. \\ ${ }^{2}$ Department of Physics and Astronomy, California State University, Long Beach, CA \\ 90840, United States.
}

\section{Simulation movies}

We present movies of the $8_{1}$ knot untying in planar elongational flows of varying strengths $(\mathrm{Wi}=1, \mathrm{Wi}=2$, $\mathrm{Wi}=3$ and $\mathrm{Wi}=4)$. All videos are written at 14 frames per second. The time elapsed between each frame is $30 l^{2} \zeta / k_{b} T$. The unknotted regions are colored in gray, the knotted regions are colored in red and the partially knotted regions are colored in blue.

*pdoyle@mit.edu 


\section{Detailed untying pathways for the $8_{1}$ knot}

As discussed in Section 2.2 of the main text, the way in which the $8_{1}$ knot unties depends on the conformational state it is in when a chain end is reached. In the $\mathrm{S}_{+}$state, the knot will completely untie to the $0_{1}$ knot; in the S- state, it will partially untie to the $6_{1}$ knot, followed by the $4_{1}$ knot and the $0_{1}$ knot, assuming no conformational rearrangement of the partially untied knots (Fig. S1). A 81 knot in both $\mathrm{D}_{+}$and $\mathrm{D}_{-}$ states will partially untie to the $6_{1}$ knot, followed by either the $0_{1}$ knot for the $\mathrm{D}_{+}$state or the $4_{1}$ knot and then the $0_{1}$ knot for the D_ state (Fig. S2).

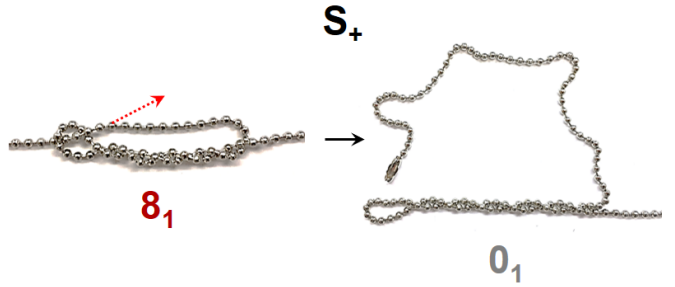

S.

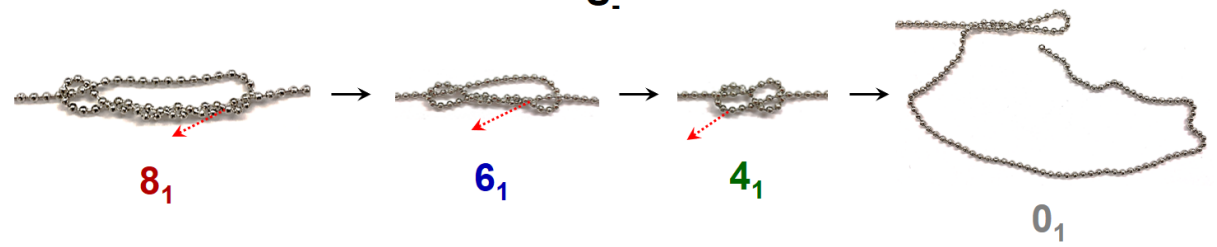

Figure S1: Illustration depicting the untying of a $8_{1}$ knot from the $\mathrm{S}_{+}$and $\mathrm{S}_{-}$states. The dashed red arrow indicates the direction in which the chain end loops through the knot during the untying process.



Figure S2: Illustration depicting the untying of a $8_{1}$ knot from the $\mathrm{D}_{+}$and $\mathrm{D}_{-}$states. The dashed red arrow indicates the direction in which the chain end loops through the knot during the untying process. 


\section{Chain tension profiles}

Fig. S3a shows the tension profiles of chains with $N=300$ beads in elongational flows at different Wi. The tension profile of a chain in an elongational flow is approximately quadratic, with maximum tension at the center of the chain and zero tension at the chain ends. Fig. S3b shows the tension profiles of chains with $N=300-600$ beads in an elongational flow at $\mathrm{Wi}=1.5$. The different chains, being at the same Wi, have the same mean tension.

(a)

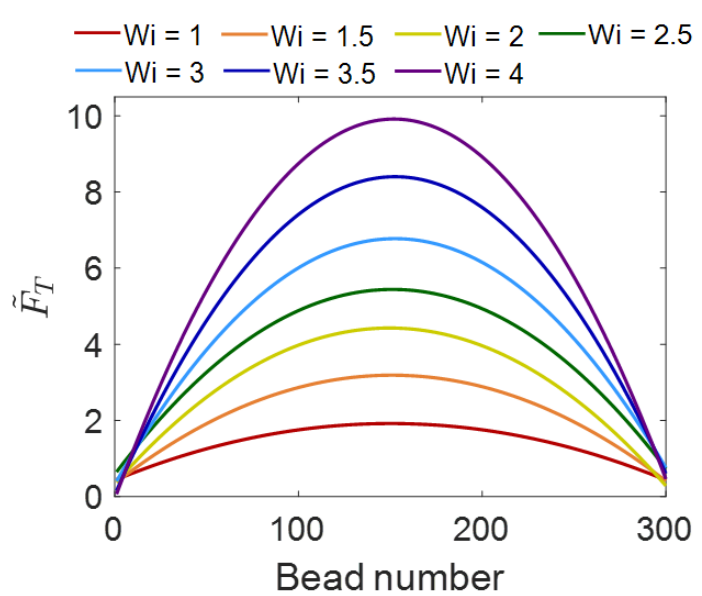

(b)

$$
W i=1.5
$$

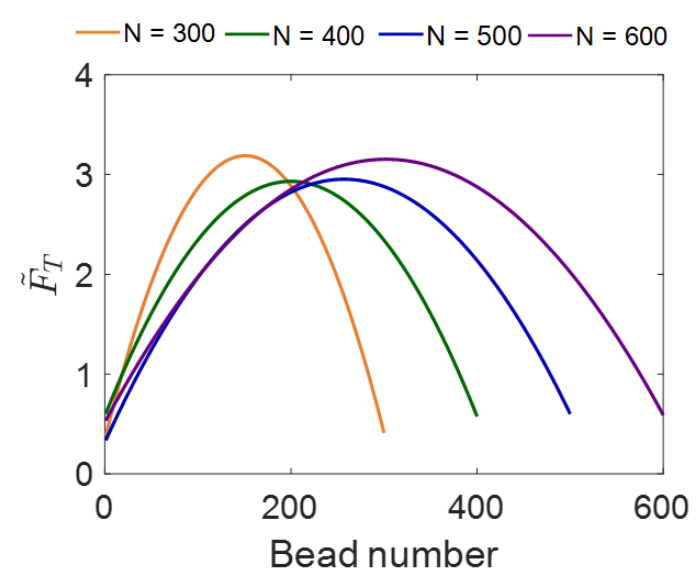

Figure S3: (a) Tension profiles of chains with $N=300$ beads in elongational flows at different Wi. (b) Tension profiles of chains with $N=300-600$ beads in elongational flow at $\mathrm{Wi}=1.5$. 


\section{Distribution of knot conformational states with different initial states}

Fig. S4 shows the distributions of conformational states for the $8_{1}$ knot immediately following chain equilibration in elongational flows of different strengths, for knots initiated in the $\mathrm{S}$ and $\mathrm{D}$ states. We observe that the distributions of knot conformational states at each Wi are similar despite the knot being initiated in different states, hence the knots studied in this work are fully equilibrated and the results are independent of the initial conformational state.
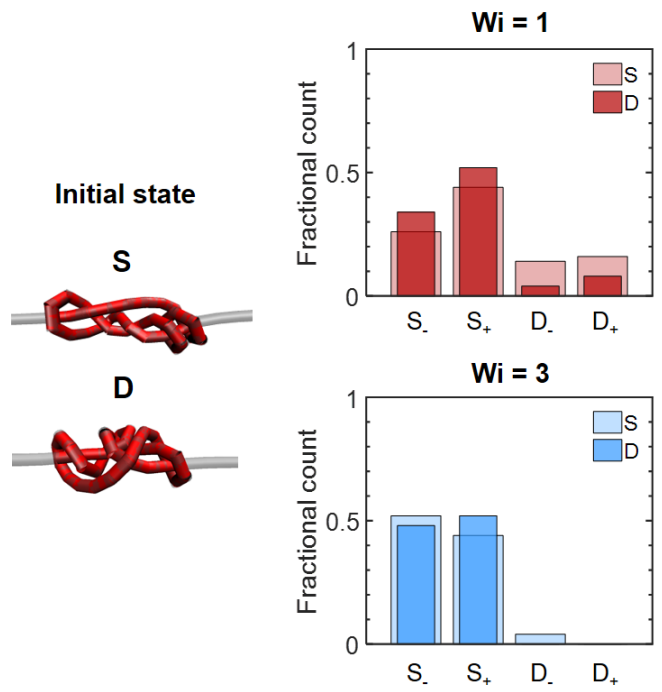

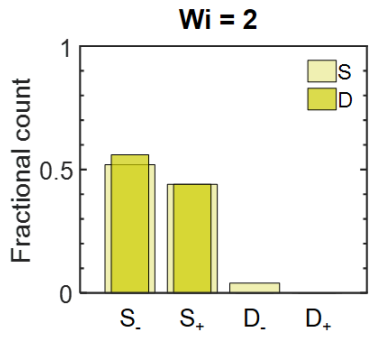

Wi $=4$

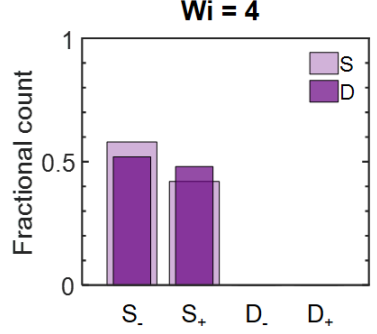

Figure S4: Distributions of conformational states for the $8_{1}$ knot at the center of equilibrated chains in elongational flows of varying strengths, with the knots initially tied in either the S or D state. 


\section{Rearrangement of conformational states for the $8_{1}$ knot}

The different conformational states of the $8_{1}$ knot are accessible from one another via rotation of the loop about the clasp of the knot, which involves moving a twist from one end of the knot to the other. Considering a 81 knot that eventually moves off the left side of the chain (Fig. S5a), the knot can rearrange from its initial $\mathrm{S}_{+}$state into the $\mathrm{D}_{+}$state, followed by the $\mathrm{D}_{-}$state and then the $\mathrm{S}_{-}$state. Fig. S5b shows an example of how rotation of certain strands within the knot with respect to others can lead to the rearrangement of conformational states. See Movie S5 for demonstration with a macroscale chain, with a bead initially part of the clasp of the knot colored white to show how the beads move during the conformational state rearrangement. In Fig. S5c, we show a representative trajectory of a $8_{1}$ knot that goes through the primary net rearrangement pathway in an elongational flow at $\mathrm{Wi}=1.5$.

(a)

(a)

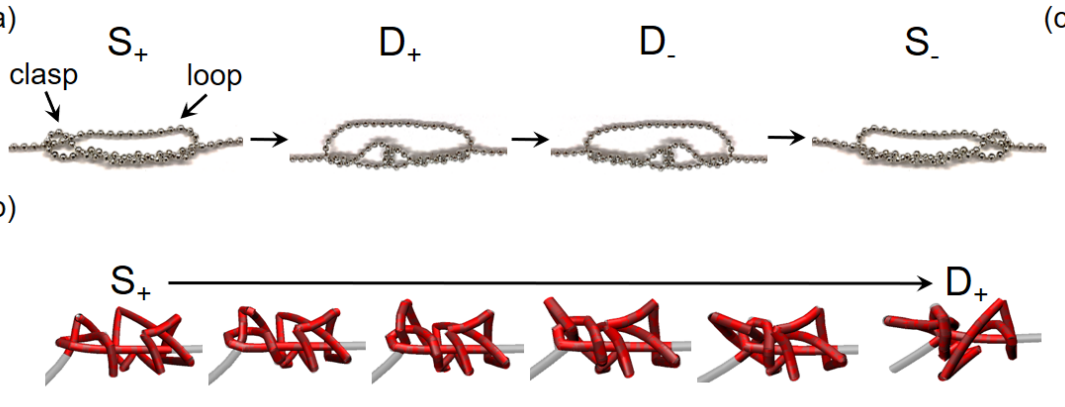

(c)

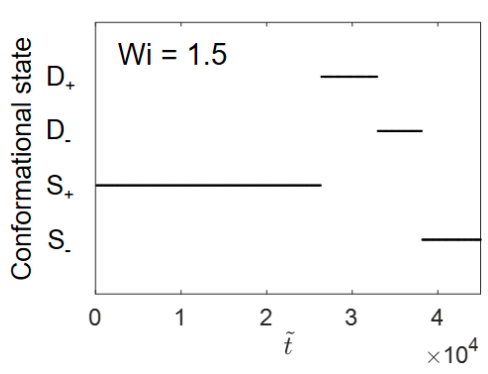

Figure S5: (a) Images of macroscale chains showing the change in conformational states as the $8_{1}$ knot traverses the primary net rearrangement pathway. (b) Simulation snapshots of the $8_{1}$ knot undergoing conformational state rearrangement from the $S_{+}$to $D_{+}$state. (c) Example of a $8_{1}$ knot undergoing the primary net rearrangement pathway in an elongational flow at $\mathrm{Wi}=1.5$. 


\section{Knot untying time}

Fig. S6 shows the mean knot untying time for the $8_{1}$ knot on chains in elongational flows as a function of

Wi. We observe an increase in the mean knot untying time from $\mathrm{Wi}=1$ to $\mathrm{Wi}=1.5-2$, followed by a decrease in untying time, indicating the transition from a diffusion-driven to convection-driven unknotting process.

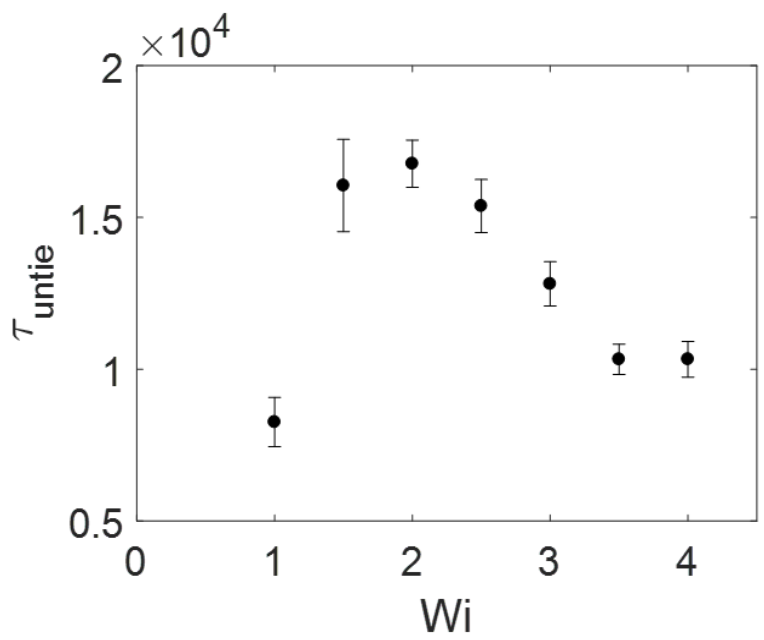

Figure S6: Mean knot untying time $\tau_{\text {untie }}$ for the $8_{1}$ knot on chains in elongational flows as a function of Wi. Each data point is an average over 50 simulation runs. Error bars represent standard error of the mean. 


\section{Frequency of knot untying pathways}

\section{Knot $7_{2}$}

\begin{tabular}{|c|c|c|c|}
\hline & $7_{2} \rightarrow 5_{2} \rightarrow 3_{1} \rightarrow 0_{1}$ & $7_{2} \rightarrow 5_{2} \rightarrow 0_{1}$ & $7_{2} \rightarrow 0_{1}$ \\
\hline $\mathrm{Wi}=3$ & $61.2 \%$ & $2.0 \%$ & $36.7 \%$ \\
$\tilde{F}_{T}=3$ & $42.0 \%$ & $4.0 \%$ & $54.0 \%$ \\
\hline
\end{tabular}

Table S1: Proportion of untying pathways for chains with a $7_{2}$ knot (Fig. 7)

Knot $9_{2}$

\begin{tabular}{|c|c|c|c|c|}
\hline & $9_{2} \rightarrow 7_{2} \rightarrow 5_{2} \rightarrow 3_{1} \rightarrow 0_{1}$ & $9_{2} \rightarrow 7_{2} \rightarrow 5_{2} \rightarrow 0_{1}$ & $9_{2} \rightarrow 7_{2} \rightarrow 0_{1}$ & $9_{2} \rightarrow 0_{1}$ \\
\hline $\mathrm{Wi}=3$ & $54.0 \%$ & $10.0 \%$ & $8.0 \%$ & $28.0 \%$ \\
$\tilde{F}_{T}=3$ & $38.0 \%$ & $8.0 \%$ & $10.0 \%$ & $44.0 \%$ \\
\hline
\end{tabular}

Table S2: Proportion of untying pathways for chains with a $9_{2}$ knot (Fig. 7)

Knot $10_{1}$

\begin{tabular}{|c|c|c|c|c|}
\hline & $10_{1} \rightarrow 8_{1} \rightarrow 6_{1} \rightarrow 4_{1} \rightarrow 0_{1}$ & $10_{1} \rightarrow 8_{1} \rightarrow 6_{1} \rightarrow 0_{1}$ & $10_{1} \rightarrow 8_{1} \rightarrow 0_{1}$ & $10_{1} \rightarrow 0_{1}$ \\
\hline $\mathrm{Wi}=3$ & $78.0 \%$ & $4.0 \%$ & $4.0 \%$ & $14.0 \%$ \\
$\tilde{F}_{T}=3$ & $30.0 \%$ & $2.0 \%$ & $4.0 \%$ & $64.0 \%$ \\
\hline
\end{tabular}

Table S3: Proportion of untying pathways for chains with a $10_{1}$ knot (Fig. 7) 